\title{
Lógica da Dedutibilidade: o axioma modal B e adjunções
}

\author{
Deductibility Logic: the modal axiom B and adjunction
}

\author{
Hércules de Araujo Feitosa \\ Departamento de Matemática, Faculdade de Ciências, Unesp \\ hercules.feitosa@unesp.br \\ ORCID: 0000-0003-0023-4192
}

\author{
Marcelo Reicher Soares \\ Departamento de Matemática, Faculdade de Ciências, Unesp \\ reicher.soares@unesp.br \\ ORCID:0000-0002-1996-5350
}

\author{
Cristiane Alexandra Lázaro \\ Departamento de Matemática, Faculdade de Ciências, Unesp \\ cristiane.lazaro@unesp.br \\ ORCID: 0000-0002-0260-6976
}

\begin{abstract}
Resumo. A lógica da dedutibilidade, ou lógica TK, formaliza no ambiente proposicional a definição do operador de consequência de Tarski. Neste processo de formalização da noção de dedutibilidade, o sistema lógico gerado, a lógica TK, tem um caráter modal para o conceito de dedução. Ela estende a lógica proposicional clássica por meio de um operador unário que retrata, na linguagem da lógica, o operador de consequência de Tarski. A lógica TK tem como modelo algébrico as TK-álgebras e como modelo topológico/conjuntista os espaços quase topológicos, ou espaços de Tarski. Os operadores modais da lógica TK, na sua contraparte topológica, estão associados aos conceitos de fecho e interior, porém estes espaços não coincidem com os usuais espaços topológicos. Iniciamos com a apresentação destas noções. Por outro lado, as conexões de Galois, que têm sua origem motivada na Teoria de Galois, são obtidas a partir dos pares de Galois, que atuam em estruturas de ordem. Flexões nos sentidos em que as ordens entre estas estruturas se aplicam geram os pares de Galois. Num segundo momento, apresentamos estas noções. Inicialmente, constatamos que os operadores de interior e fecho definidos sobre os espaços quase topológicos não determinam algum par de Galois. Mas o que fariam estes operadores caírem na condição de algum par de Galois? Quando analisado no contexto lógico, vislumbramos que a inclusão do conhecido axioma modal $\mathbf{B}$ à lógica TK nos daria um tal par. Assim, a contrapartida de tal axioma, no contexto dos espaços quase topológicos, nos levou à obtenção de uma adjunção a partir dos seus respectivos operadores de fecho e interior.
\end{abstract}


Palavras-chave. Matemática discreta. Espaços quase topológicos. Lógica TK. Pares de Galois. Adjunção.

\begin{abstract}
Logic TK formalizes in the propositional environment the notion of the Tarski consequence operator. Because that it is called the logic of deductibility. In this process of formalizing the notion of deductibility, the logic obtained has a modal character. It is obtained from the classical propositional logic plus an unary operator relative to the Tarski operator. Logic TK has as its algebraic model the TK-algebras and as topological / set theoretic model the quasi-topological spaces, or Tarski spaces. The modal operators of logic TK are associated, in their topological counterpart, with the concepts of closure and interior. Galois connections, in turn, are originated from Galois theory. They are obtained from the Galois pairs, which act over order structures with changes in the in the orders that define these structures. In a first approach, we find that the interior and close operators of almost topological spaces do not determine a Galois pair. When analysed in the logical context, if we include the well-known modal axiom $\mathbf{B}$ on the logic TK the operators determine an adjunction that is a Galois pair. The counterpart of such procedure, in almost topological context, led us to reach an adjunction from the closure and interior operators.
\end{abstract}

Keywords. Discrete mathematics. Almost topological spaces. Logic TK. Galois pairs. Adjunction.

Mathematics Subject Classification (MSC): primary 03B22; secondary 03B45.

\title{
1 Introdução
}

Espaços de Tarski caracterizam o conceito de dedutibilidade e, desse modo, são modelos naturais para a lógica da dedutibilidade TK.

Neste artigo, destacamos o comportamento de um espaço de Tarski ao introduzirmos um particular e notável axioma modal na lógica TK. Balizamos estas ações a partir do conceito algébrico de adjunção que é um particular par de Galois.

Para darmos os detalhes da construção pretendida, iniciamos com a apresentação dos espaços de Tarski, que podem ser vistos em [3], [1] e [4].

Então apresentamos as TK-álgebras que podem ser encontradas em [8] e [3]. Como correspondente lógico proposicional das TK-álgebras, temos a lógica TK, que é introduzida na seção seguinte, como em [3] e [4].

Para os pares de Galois, fazemos uma outra seção com sua definição e algumas propriedades importantes para o desenvolvimento posterior, conforme em [2], [6], [9], [10] e 
[11]. Adjunções são casos específicos de pares de Galois. Para detalhes podemos mencionar ainda [5].

Espaço quase topológico é a versão topológica dos espaços de Tarski. De fato, é uma maneira alternativa de se definir os espaços de Tarski, trocando a intuição da noção de dedução pela noção topológica de fecho [3]. Nos espaços quase topológicos temos operadores de fecho e interior que, como veremos, não constituem pares de Galois.

\section{Espaços de Tarski}

Os espaços de Tarski surgem da busca por uma caracterização, bastante geral, do que um sistema formal deveria contemplar para ser considerado como uma lógica (ver [3] e [4]).

Definição 1. Operador de consequência sobre $E$ é uma função ${ }^{-}: \mathcal{P}(E) \rightarrow \mathcal{P}(E)$ tal que, para todos $A, B \subseteq E$, valem:

(i) $A \subseteq \bar{A}$

(ii) $A \subseteq B \Rightarrow \bar{A} \subseteq \bar{B}$

(iii) $\overline{\bar{A}} \subseteq \bar{A}$.

Segue, de (i) e (iii), que vale a igualdade $\overline{\bar{A}}=\bar{A}$, para cada $A \subseteq E$.

Definição 2. Espaço de Tarski (ou um sistema dedutivo de Tarski ou um espaço de fecho) é um par $\left(E,^{-}\right)$de modo que E é um conjunto $e^{-}$é um operador de consequência sobre E.

Definição 3. Se $\left(E,^{-}\right)$é um espaço de Tarski, então o conjunto A é fechado em $\left(E,^{-}\right)$ se $\bar{A}=A$, e A é aberto quando o seu complemento relativo a $E$, denotado por $A^{C}, e ́$ fechado em $\left(E,^{-}\right)$.

Proposição 1. Em cada espaço de Tarski $\left(E,^{-}\right)$, qualquer intersecção de conjuntos fechados é ainda um conjunto fechado.

Segue das definições que $\bar{\emptyset}$ e $E$ correspondem ao menor e ao maior conjuntos fechados, respectivamente, associados ao operador ${ }^{-}$.

Espaços quase topológicos são generalizações das estruturas topológicas dadas pela eliminação de algumas propriedades.

Definição 4. Espaço quase topológico é um par $(E, \Omega)$ em que E é um conjunto, $\Omega \subseteq \mathcal{P}(E) e:$

(i) para qualquer $B \subset \Omega$, segue que $\cup B \in \Omega$. 
Notemos que se $B=\emptyset$, em (i), então $\cup B=\cup \emptyset=\emptyset \in \Omega$

Definição 5. A coleção $\Omega$ é denominada quase topologia e cada membro de $\Omega$ é um aberto de $(E, \Omega)$. Um conjunto $A \in \mathcal{P}(E)$ é um fechado quando o seu complemento relativo a $E$, denotado por $A^{C}$, é um aberto de $(E, \Omega)$.

Segue destas definições que uma união qualquer de abertos de $(E, \Omega)$ é ainda um aberto de $(E, \Omega)$.

Proposição 2. Em todo espaço quase topológico $(E, \Omega)$ o conjunto $\emptyset$ é um aberto e E é um fechado.

Proposição 3. Em todo espaço quase topológico $(E, \Omega)$, qualquer intersecção de fechados é um fechado.

Definição 6. Se $(E, \Omega)$ é um espaço quase topológico, então o fecho de A é o conjunto: $\bar{A}=\cap\left\{X: A \subseteq X\right.$ e $\left.X^{C} \in \Omega\right\}$. O interior de A é o conjunto: $\AA=\cup\{X \subseteq E: X \subseteq$ A e $X \in \Omega\}$.

Proposição 4. Se $(E, \Omega)$ é um espaço quase topológico e $A \subseteq E$, então $\bar{A}$ é fechado e $\AA$ é aberto.

Naturalmente, poderíamos definir espaço quase topológico a partir dos fechados, como no contexto topológico.

Proposição 5. Seja $(E, \Omega)$ um espaço quase topológico. Para todos $A, B \subseteq E$, valem:

(i) $\AA \subseteq A \subseteq \bar{A}$

(ii) $\stackrel{̊}{\AA}=\AA$

(iii) $\overline{\bar{A}}=\bar{A}$

(iv) $A \subseteq B \Rightarrow \stackrel{\circ}{A} \subseteq \stackrel{\circ}{B}$

(v) $A \subseteq B \Rightarrow \bar{A} \subseteq \bar{B}$.

Também poderíamos definir o espaço quase topológico a partir da operação de interior e dela definirmos o fecho de Tarski.

Definição 7. Operador de interior sobre E é uma função ${ }^{\circ} \mathcal{P}(E) \rightarrow \mathcal{P}(E)$ tal que, para todos $A, B \subseteq E$, valem:

(i) $\AA \subseteq A$

(ii) $A \subseteq B \Rightarrow \stackrel{\circ}{A} \stackrel{\circ}{B}$

(iii) $\AA \subseteq \stackrel{\circ}{A}$.

Agora mostramos a relação entre espaços de Tarski e espaços quase topológicos. 
Proposição 6. Sejam $(E, \Omega)$ um espaço quase topológico $e^{-}$a operação de fecho em $(E, \Omega)$. Então o par $\left(E,^{-}\right)$é um espaço de Tarski.

Por outro lado, se $\left(E,^{-}\right)$é um espaço de Tarski, ao considerarmos $\Psi=\{X \subseteq E$ : $X=\bar{X}\}$, o conjunto dos fechados de $\left(E,{ }^{-}\right)$, então segue a proposição seguinte.

Proposição 7. Se $\left(E,^{-}\right)$é um espaço de Tarski, então $(E, \Omega)$ é um espaço quase topológico em que $X \in \Omega \Leftrightarrow X^{C} \in \Psi$.

Segue das duas proposições anteriores que, para cada espaço de Tarski, podemos definir, de modo natural, um espaço quase topológico e, para cada espaço quase topológico, podemos definir um espaço de Tarski.

Definição 8. Um espaço quase topológico $(E, \Omega)$ é O-fechado quando:

(iv) $\bar{\emptyset}=\emptyset$.

Definição 9. Um espaço topológico $(E, \Omega)$ é um espaço quase topológico O-fechado tal que:

(v) $\overline{A \cup B}=\bar{A} \cup \bar{B}$.

Definição 10. Um espaço de Tarski $\left(E,^{-}\right)$é vácuo quando $\bar{\emptyset}=\emptyset$.

Deste modo, os espaços topológicos são exemplos de espaços quase topológicos 0fechados. Como o conceito de consequência é essencial à Lógica e naquele contexto são relevantes os conjuntos de consequências, os quais não devem ser em geral vazios, do ponto de vista lógico interessam os espaços de Tarski não vácuos.

\section{TK-álgebras}

Nesta seção apresentamos as TK-álgebras e na seguinte a lógica proposicional TK. Detalhes sobre os dois sistemas podem ser encontrados em [3].

A definição de TK-álgebra introduz a noção de operador de consequência, da Definição 1, no ambiente algébrico e a lógica TK dá a formalização no contexto lógico.

Definição 11. Uma TK-álgebra é uma sêxtupla $\mathcal{A}=\langle A, 0,1, \vee, \sim, \bullet\rangle$ de maneira que $\langle A, 0,1, \vee, \sim\rangle$ é uma álgebra de Boole e $\bullet$ é um novo operador, chamado de operador de Tarski, para o qual valem:

(i) $a \vee \bullet a=\bullet a$

(ii) $\bullet a \vee \bullet(a \vee b)=\bullet(a \vee b)$

(iii) $\bullet(\bullet a)=\bullet a$. 
Como as TK-álgebras estão no contexto das álgebras de Boole, o item (i) da definição acima afirma que, para cada $a \in A, a \leq \bullet a$ e o item (ii) que se $a \leq b$, então $\bullet a \leq \bullet$.

Exemplo 1. A álgebra de Boole conjuntista com $A=\mathcal{P}(X)$, quando $X \neq \emptyset$, munida do operador que para todo $a \in A$, associa $\bullet a=$ a é uma TK-álgebra.

A função identidade é um operador trivial de Tarski.

Exemplo 2. A álgebra de conjuntos $\mathcal{P}(\mathbb{R})$ com $\bullet X=X \cup\{0\}$ é uma TK-álgebra.

Exemplo 3. A álgebra de conjuntos $\mathcal{P}(\mathbb{R})$ com $\bullet X=\cap\{I \subseteq \mathbb{R}: I$ é um intervalo e $X \subseteq$ I\} é uma TK-álgebra.

Nas TK-álgebras podemos definir os seguintes operadores:

$$
\begin{aligned}
& a \longmapsto b={ }_{d f} \sim a \vee b \\
& a-b={ }_{d f} a \wedge \sim b .
\end{aligned}
$$

Proposição 8. Para toda TK-álgebra valem as seguintes condições:

(i) $\sim \bullet a \leq \sim a \leq \bullet \sim a$

(ii) $a \leq b \Rightarrow \bullet a \leq \bullet b$

(iii) $\bullet(a \wedge b) \leq \bullet a \wedge \bullet b$

(iv) $\bullet a \vee \bullet b \leq \bullet(a \vee b)$.

\section{A lógica TK}

A lógica proposicional TK é o sistema lógico construído sobre a linguagem proposicional de TK que é $L=\left\{\neg, \vee, \rightarrow, \diamond, p_{1}, p_{2}, p_{3}, \ldots\right\}$ com os seguintes axiomas e regras:

$\begin{array}{ll}(\mathrm{CPC}) & \varphi, \text { se } \varphi \text { é uma tautologia; } \\ \left(T K_{1}\right) & \varphi \rightarrow \diamond \varphi ; \\ \left(T K_{2}\right) & \diamond \bullet \rightarrow \diamond \varphi . \\ (\mathrm{MP}) & \frac{\varphi \rightarrow \psi, \varphi}{\psi} ; \\ \left(R M^{\diamond}\right) & \frac{\vdash \varphi \rightarrow \psi}{\vdash \bullet \varphi \rightarrow \diamond \psi} .\end{array}$


Este sistema lógico formaliza, agora numa linguagem proposicional, as noções essenciais do operador de consequência de Tarski, da Definição 1.

O operador da Definição 1 pressupõe um espaço de conjuntos subjacente, que corresponde aqui ao sistema Booleano das tautologias e da regra MP. As condições do operador são aqui formalizadas pelos axiomas $\left(T K_{1}\right),\left(T K_{2}\right)$ e a regra $\left(R M^{\star}\right)$.

Proposição 9. $\vdash \vee \varphi \rightarrow(\varphi \vee \psi)$.

Demonstração.
1. $\varphi \rightarrow(\varphi \vee \psi)$
Tautologia
2. $\varphi \rightarrow \diamond(\varphi \vee \psi)$
$R M \bullet$ em 1

Proposição 10. $\vdash \varphi \Rightarrow \vdash \diamond \varphi$.
1. $\varphi$
Premissa
Demonstração.
2. $\varphi \rightarrow \varphi$
$A x_{T K 1}$
3. $\varphi$
MP em 1 e 2 .

Note que esta última proposição afirma que se $\varphi$ é um teorema de TK então também é um teorema, mas se $\varphi$ é uma variável, por exemplo, então o resultado não se aplica.

Proposição 11. $\vdash \vee \varphi \vee \psi \rightarrow \diamond(\varphi \vee \psi)$.

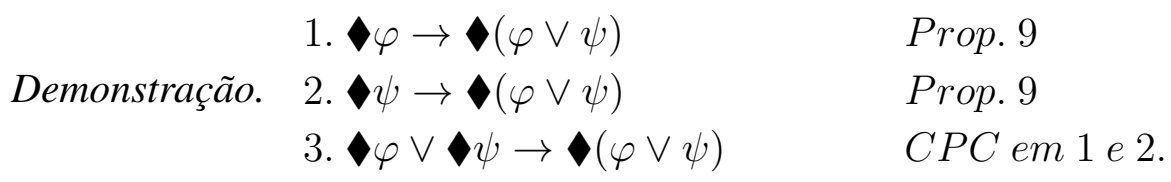

Em [3] está uma demonstração da adequação (correção e completude) de TK relativo às TK-álgebras.

Podemos definir o operador dual de $\downarrow$ da seguinte maneira:

$$
\diamond \varphi=_{d f} \neg \neg \varphi \text {. }
$$

Proposição 12. $\vdash \varphi \rightarrow \psi \Rightarrow \vdash \diamond \varphi \rightarrow \diamond \psi$.

Corolário 1. $\vdash \varphi \leftrightarrow \psi \Rightarrow \vdash \nabla \varphi \leftrightarrow \diamond \psi$.

Proposição 13. $\vdash \diamond \varphi \rightarrow \varphi$.

Proposição 14. $\vdash \diamond \varphi \rightarrow \diamond \diamond \varphi$.

Proposição 15. $\vdash \diamond(\varphi \wedge \psi) \rightarrow \diamond \varphi$. 
Corolário 2. $\vdash \diamond(\varphi \wedge \psi) \rightarrow(\diamond \varphi \wedge \diamond \psi)$.

Poderíamos, alternativamente, ter tomado o operador $\diamond$ como o operador primitivo e substituir os axiomas $T K_{1}$ e $T K_{2}$ pelos seguintes axiomas e regra de dedução:

$$
\begin{aligned}
& \left(T K_{1}^{*}\right) \diamond \varphi \rightarrow \varphi \\
& \left(T K_{2}^{*}\right) \nabla \varphi \rightarrow \diamond \diamond \varphi \\
& \left(R M^{\boxminus}\right) \frac{\vdash \varphi \rightarrow \psi}{\vdash \nabla \varphi \rightarrow \diamond \psi} .
\end{aligned}
$$

Dado um espaço quase topológico $(E, \Omega)$, o operador $\downarrow$ está associado ao fecho naquele espaço, enquanto o operador $\diamond$ está associado ao interior.

\section{Pares de Galois}

Pares de Galois são duas funções definidas entre duas estruturas de ordem com sentidos inversos, que preservam certos aspectos das ordens das duas estruturas. São generalizações das conexões de Galois, que surgiram nos trabalhos originais do famoso algebrista francês.

Nas notas seguintes, damos os detalhes. Ver, por exemplo, [2], [9] e [11].

Definição 12. Uma relação binária $\leq$ sobre um conjunto $A$ é uma ordem parcial se a relação $\leq e ́$ :

(i) reflexiva: para todo $a \in A$, vale que $a \leq a$;

(ii) antissimétrica: para todos $a, b \in A$, se $a \leq b$ e $b \leq a$, então $a=b$;

(iii) transitiva: para todos $a, b, c \in A$, se $a \leq b$ e $b \leq c$, então $a \leq c$.

Definição 13. Conjunto parcialmente ordenado (poset) é um par $\langle A, \leq\rangle$ em que A é um conjunto não vazio e $\leq$ é uma ordem parcial sobre $A$.

Definição 14. Se $f:\left(A, \leq_{A}\right) \rightarrow\left(P, \leq_{P}\right)$ é uma função entre dois conjuntos parcialmente ordenados, então:

(i) a função $f$ preserva as ordens se $a \leq_{A} b \Rightarrow f(a) \leq_{P} f(b)$;

(ii) a função $f$ inverte as ordens se $a \leq_{A} b \Rightarrow f(b) \leq_{P} f(a)$.

Definição 15. Se $f:\left(A, \leq_{A}\right) \rightarrow\left(A, \leq_{A}\right)$, então:

(i) a função $f$ é extensiva ou inflacionária se, para todo $a \in A, a \leq f(a)$;

(ii) a função $f$ é deflacionária se, para todo $a \in A, f(a) \leq a$.

Definição 16. Se $\left(A, \leq_{A}\right) e\left(P, \leq_{P}\right)$ são conjuntos parcialmente ordenados, a $\in A e$ $p \in P$ são elementos quaisquer e $f: A \rightarrow P$ e $g: P \rightarrow A$ são funções, então:

(i) o par $(f, g)$ é uma conexão de Galois se: $a \leq_{A} g(p) \Longleftrightarrow p \leq_{P} f(a)$ 
(ii) o par $(f, g)^{d}$ é uma conexão dual de Galois se: $g(p) \leq_{A} a \Longleftrightarrow f(a) \leq_{P} p$

(iii) o par $[f, g]$ é uma adjunção se: $a \leq_{A} g(p) \Longleftrightarrow f(a) \leq_{P} p$

(iv) o par $[f, g]^{d}$ é uma adjunção dual se: $g(p) \leq_{A} a \Longleftrightarrow p \leq_{P} f(a)$.

Em alguns textos, o par $[f, g]$ também é chamado de residuado.

$\operatorname{Se}\left(A, \leq_{A}\right)$ é um conjunto parcialmente ordenado, então denotaremos a ordem inversa de $\leq_{A}$ por $\leq_{A}^{o p}$ e, desse modo, $\left(A, \leq_{A}^{o p}\right)=\left(A,\left(\leq_{A}\right)^{-1}\right)$. Assim, $a \leq_{A} b \Leftrightarrow b \leq_{A}^{o p} a$.

Sejam $\left(A, \leq_{A}\right)$ e $\left(P, \leq_{P}\right)$ conjuntos parcialmente ordenados e $f: A \rightarrow P$ e $g: P \rightarrow$ $A$ funções:

(i) Se $(f, g)$ é uma conexão de Galois, então $(g, f)$ também é uma conexão de Galois.

(ii) $\mathrm{Se}(f, g)^{d}$ é uma conexão dual de Galois, então $(g, f)^{d}$ também é uma conexão dual de Galois.

(iii) Se $[f, g]$ é uma adjunção, então $[g, f]^{d}$ é uma adjunção dual.

(iv) Se $[f, g]^{d}$ é uma adjunção dual, então $[g, f]$ é uma adjunção.

Se $(f, g)$ é uma conexão de Galois para $\left(A, \leq_{A}\right)$ e $\left(P, \leq_{P}\right)$, então:

(i) $(f, g)^{d}$ é uma conexão dual de Galois para $\left(A, \leq_{A}^{o p}\right)$ e $\left(P, \leq_{P}^{o p}\right)$.

(ii) $[f, g]$ é uma adjunção para $\left(A, \leq_{A}\right)$ e $\left(P, \leq_{P}^{o p}\right)$.

(iii) $[f, g]^{d}$ é uma adjunção dual para $\left(A, \leq_{A}^{o p}\right)$ e $\left(P, \leq_{P}\right)$.

A seguir, destacamos as adjunções, como um caso de par de Galois. Demonstramos vários resultados sobre as adjunções. Estes resultados, com as devidas particularidades, podem ser aplicados aos demais pares de Galois.

\section{Adjunções}

Nesta seção, tomamos um dos pares de Galois, as adjunções, e mostramos muitas propriedades das adjunções.

Em alguns textos sobre os pares de Galois, as adjunções são chamadas de Conexões de Galois, como em [11]. Mas a nossa escolha do nome de Conexão de Galois está de acordo com a original Teoria de Galois.

Seguimos a apresentação de [2] e [10].

Definição 17. Dados dois conjuntos parcialmente ordenados $\left(A, \leq_{A}\right)$ e $\left(P, \leq_{P}\right)$ e as funções $f: A \rightarrow P$ e $g: P \rightarrow A$, então o par $[f, g]$ é uma adjunção de Galois se para todo a $\in$ A e todo $p \in P$ temos que:

$$
a \leq_{A} g(p) \Longleftrightarrow f(a) \leq_{P} p .
$$


Em geral, não indicamos as ordens $\leq_{A} \mathrm{e} \leq_{P}$, pois o contexto permite dizer sobre qual conjunto tratamos da ordem em questão.

A proposição seguinte nos dá condições para termos uma adjunção.

Proposição 16. Sejam $\left(A, \leq_{A}\right) e\left(P, \leq_{P}\right)$ duas ordens parciais, $f: A \rightarrow P$ e $g: P \rightarrow A$ funções, $a, b \in A$ e $p, q \in P$. Então o par $[f, g]$ é uma adjunção se, e somente se, valem as condições:

(i) $a \leq g(f(a))$

(ii) $f(g(p)) \leq p$

(iii) $a \leq b \Rightarrow f(a) \leq f(b)$

(iv) $p \leq q \Rightarrow g(p) \leq g(q)$.

Demonstração. $(\Rightarrow)$ (i) Como $f(a) \leq f(a)$ e $[f, g]$ é uma adjunção, então $f(a) \leq$ $f(a) \Leftrightarrow a \leq g(f(a))$. Logo, $a \leq g(f(a))$.

(ii) Como $g(p) \leq g(p)$ e $[f, g]$ é uma adjunção, então $g(p) \leq g(p) \Leftrightarrow f(g(p)) \leq p$. Logo, $f(g(p)) \leq p$.

(iii) Seja $a \leq b$. Por (i), $b \leq g(f(b))$ e, então, $a \leq g(f(b))$. Desde que $[f, g]$ é uma adjunção, então $a \leq g(f(b)) \Leftrightarrow f(a) \leq f(b)$. Logo, $f(a) \leq f(b)$

(iv) Seja $p \leq q$. Por (ii), $f(g(p)) \leq p$ e, então, $f(g(p)) \leq q$. Desde que $[f, g]$ é uma adjunção, então $f(g(p)) \leq q \Leftrightarrow g(p) \leq g(q)$. Logo, $g(p) \leq g(q)$.

$(\Leftarrow)$ Ida: $a \leq g(p) \Rightarrow f(a) \leq f(g(p)) \Rightarrow f(a) \leq p$.

Volta: $f(a) \leq p \Rightarrow g(f(a)) \leq g(p) \Rightarrow a \leq g(p)$.

Temos então outra maneira de definirmos uma adjunção. O par $[f, g]$ é uma adjunção se as funções $f$ e $g$ preservam as ordens e as compostas $g \circ f$ e $f \circ g$ são, respectivamente, inflacionária e deflacionária.

Proposição 17. Se o par $[f, g]$ é uma adjunção para as ordens parciais $\left(A, \leq_{A}\right) e\left(P, \leq_{P}\right.$ ), então: $f(a)=f(g(f(a)))$ e $g(b)=g(f(g(b)))$.

Demonstração. De (i), $a \leq g(f(a))$ e então, por (iii), $f(a) \leq f(g(f(a)))$. Todavia, por (ii), $f(g(f(a))) \leq f(a)$. Assim, $f(a)=f(g(f(a)))$. A outra igualdade sai de modo análogo.

Proposição 18. Se o par $[f, g]$ é uma adjunção para $\left(A, \leq_{A}\right)$ e $\left(P, \leq_{P}\right)$, então as duas composições $g \circ f$ e $f \circ g$ são operadores de Tarski (fecho) e de interior, respectivamente, sobre $A$ e $P$. 
Demonstração. Por (i), para todo $a \in A, a \leq g(f(a))$. Agora, se $a \leq b$, então $f(a) \leq$ $f(b)$ e, daí, $g(f(a)) \leq g(f(b))$. Como, da proposição anterior, $f(g(f(a)))=f(a)$, então $g(f(g(f(a))))=g(f(a))$.

Logo, $g \circ f$ é um operador de Tarski sobre $A$.

Para a composição $f \circ g$, a justificativa é análoga.

Exemplo 4. Dados dois conjuntos $E$ e $F$, seja $R$ uma relação de $E$ em $F$, isto é, $R \subseteq$ $E \times F$ e consideremos então as seguintes relações de ordem $(\mathcal{P}(E), \subseteq)$ e $(\mathcal{P}(F), \subseteq)$.

Agora, definamos as seguintes funções: $f: \mathcal{P}(E) \rightarrow \mathcal{P}(F)$ dada por $A^{f}=\{y \in F$ : $(\exists x \in A)(x R y)\} ;$ e $g: \mathcal{P}(F) \rightarrow \mathcal{P}(E)$ dada por $B^{g}=\{x \in E:(\forall y)(x R y \rightarrow y \in B)\}$.

Mostraremos que $[f, g]$ é uma adjunção. Para tanto, devemos observar que (i) $A \subseteq\left(A^{f}\right)^{g}$; (ii) $\left(B^{g}\right)^{f} \subseteq B$; (iii) $A_{1} \subseteq A_{2} \Rightarrow A_{1}^{f} \subseteq A_{2}^{f}$; (iv) $B_{1} \subseteq B_{2} \Rightarrow B_{1}^{g} \subseteq B_{2}^{g}$, conforme Proposição 16.

(i) Suponhamos que $A \nsubseteq\left(A^{f}\right)^{g}$. Então existe $x \in A$ tal que $x \notin\left(A^{f}\right)^{g}$. Daí, não é o caso que $(\forall y)\left(x R y \rightarrow y \in A^{f}\right)$. Logo, existe y tal que $x R y$ e $y \notin A^{f}$. Se $y \notin A^{f}$, então não é o caso que existe $x$ pertencente a A tal que $x$ Ry, o que contradiz a afirmação acima.

(ii) Se $y \in\left(B^{g}\right)^{f}$, então existe $x \in B^{g}$ tal que $x R y$. Agora, se $x$ Ry e $x \in B^{g}$, então $y \in$ B. Logo, $\left(B^{g}\right)^{f} \subseteq B$.

(iii) Consideremos que $A_{1} \subseteq A_{2}$. Se $y \in A_{1}^{f}$, então $\left(\exists x \in A_{1}\right)(x R y)$. Da hipótese, temos que $\left(\exists x \in A_{2}\right)(x R y)$ e, portanto, $y \in A_{2}^{f}$. Logo $A_{1}^{f} \subseteq A_{2}^{f}$.

(iv) Consideremos que $B_{1} \subseteq B_{2}$. Se $x \in B_{1}^{g}$, então $(\forall y)\left(x R y \rightarrow y \in B_{1}\right)$. Da hipótese, temos que $(\forall y)\left(x R y \rightarrow y \in B_{2}\right)$ e, portanto, $x \in B_{2}^{g}$. Logo $B_{1}^{g} \subseteq B_{2}^{g}$.

Exemplo 5. Como um caso particular do exemplo anterior, consideremos que $E=F e$ $R$ é uma ordem parcial $\leq$ sobre $E$.

Então $f: \mathcal{P}(E) \rightarrow \mathcal{P}(E)$ é definida por $A^{f}=\{y \in E:(\exists x \in A)(x \leq y)\}$, que consiste de todos os elementos de A e elementos de E que majoram algum elemento de $A$; e g $: \mathcal{P}(E) \rightarrow \mathcal{P}(E)$ é definida por $B^{g}=\{x \in E:(\forall y)(x \leq y \rightarrow y \in B)\}$, que consiste de todos os elementos de $B$ e elementos de $E$ que minoram qualquer elemento de $B$.

Exemplo 6. Consideremos uma função $h: E \rightarrow F$ e as relações de ordem $(\mathcal{P}(E), \subseteq) e$ $(\mathcal{P}(F), \subseteq)$.

Definamos então a imagem direta via $h$ por $f: \mathcal{P}(E) \rightarrow \mathcal{P}(F)$ com $A^{f}=\{y \in$ $F: x \in A$ e $h(x)=y\}$; e a imagem inversa via $h$ por $g: \mathcal{P}(F) \rightarrow \mathcal{P}(E)$ com $B^{g}=\{x \in E: y \in B$ e $h(x)=y\}$.

Mostraremos que $[f, g]$ é uma adjunção.

Certamente as funções $f$ e g preservam as ordens. 
Se $y \in\left(B^{g}\right)^{f}$, então existe $x \in B^{g}$ tal que $h(x)=y$. Agora, como $h(x)=y e$ $x \in B^{g}$, então $y \in B$. Logo, $\left(B^{g}\right)^{f} \subseteq B$.

Se $x \in A$, como hé função, então para $y=h(x)$ tem-se que $y \in A^{f}$. Daí, segue que $x \in\left(A^{f}\right)^{g}$, ou seja, $A \subseteq\left(A^{f}\right)^{g}$.

Proposição 19. Se o par $[f, g]$ é uma adjunção para os reticulados (definidos em [2] $e$ [7]) $(A, \wedge, \vee) e(P, \wedge, \vee)$, então valem:

(i) $f(x \vee y)=f(x) \vee f(y)$

(ii) $g(x \wedge y)=g(x) \wedge g(y)$.

Demonstração. (i) Como $x \leq x \vee y$ e $y \leq x \vee y$, então $f(x) \leq f(x \vee y)$ e $f(y) \leq f(x \vee y)$, donde segue que $f(x) \vee f(y) \leq f(x \vee y)$.

Por outro lado, $f(x) \leq f(x) \vee f(y)$ e $f(y) \leq f(x) \vee f(y)$, donde segue que $x \leq$ $g(f(x) \vee f(y))$ e $y \leq g(f(x) \vee f(y))$. Assim, $x \vee y \leq g(f(x) \vee f(y)) \Rightarrow f(x \vee y) \leq$ $f(x) \vee f(y)$.

(ii) Similar.

Proposição 20. Se $\left[f, g_{1}\right] e\left[f, g_{2}\right]$ são adjunções para $\left(A, \leq_{A}\right) e\left(P, \leq_{P}\right)$, então: $g_{1}=g_{2}$. $\mathrm{Se}\left[f_{1}, g\right] e\left[f_{2}, g\right]$ são adjunções para $\left(A, \leq_{A}\right) e\left(P, \leq_{P}\right)$, então: $f_{1}=f_{2}$.

Demonstração. Dado $p \in P$, como $g_{1}$ e $g_{2}$ são funções, então estão definidos $g_{1}(p)$ e $g_{2}(p)$. Da definição de adjunção segue que: $g_{1}(p) \leq g_{2}(p) \Leftrightarrow f\left(g_{1}(p)\right) \leq p$ e $g_{2}(p) \leq g_{1}(p) \Leftrightarrow f\left(g_{2}(p)\right) \leq p$. Como o lado direito das duas equivalências sempre é vá lido para todo $p$ pertencente a $P$, devido às hipóteses consideradas (adjunções consideradas) e à Proposição 16 (ii), resulta que o lado esquerdo de ambas as equivalências são válidos e, portanto, $g_{1}=g_{2}$.

A outra condição é semelhante a esta.

Proposição 21. Se o par $[f, g]$ é uma adjunção para $\left(A, \leq_{A}\right)$ e $\left(P, \leq_{P}\right)$, então valem:

(i) $a \in g(P) \Leftrightarrow g(f(a))=a$

(ii) $p \in f(A) \Leftrightarrow f(g(p))=p$

(iii) $f(A)=f(g(P))$

(iv) $g(P)=g(f(A))$.

Demonstração. (i) $(\Rightarrow)$ Se $a \in g(P)$, então existe $p \in P$ tal que $g(p)=a$. Daí, $g(f(a))=g(f(g(p)))=g(p)=a .(\Leftarrow)$ Se $g(f(a))=a$, então para $b=f(a)$ temos que $g(b)=a$ e, portanto, $a \in g(P)$.

(ii) Similar a (i).

(iii) Se $p \in f(A)$, por (ii), $p=f(g(p))$ e, portanto, $p \in f(g(P))$. Logo $f(A) \subseteq$ $f(g(P))$. Agora, se $p \in f(g(P))$, então $p=f(a)$ para algum $a \in g(P) \subseteq A$. Assim, $p \in f(A)$ e $f(g(P)) \subseteq f(A)$. 
(iv) Similar a (iii).

Assim, cada ponto $a \in g(P)$ é ponto fixo da função $g \circ f$ e cada ponto $p \in f(A)$ é ponto fixo da função $f \circ g$.

Proposição 22. Se o par $[f, g]$ é uma adjunção para $\left(A, \leq_{A}\right)$ e $\left(P, \leq_{P}\right)$, então:

(i) $f(a)=\min \{p \in P: a \leq g(p)\}$

(ii) $g(p)=\max \{a \in A: f(a) \leq p\}$.

Demonstração. (i) Dado $a \in A$, como $a \leq g(f(a))$, então $f(a) \in\{p \in P: a \leq g(p)\}$. Agora, se $c \in\{p \in P: a \leq g(p)\}$, então $a \leq g(c)$ e daí $f(a) \leq f(g(c))$ e, portanto, $f(a) \leq c$. Logo, $f(a)=\min \{p \in P: a \leq g(p)\}$.

(ii) Dado $p \in P$, como $f(g(p)) \leq p$, então $g(p) \in\{a \in A: f(a) \leq p\}$. Agora, se $c \in\{a \in A: f(a) \leq p\}$, então $f(c) \leq p$ e daí $g(f(c)) \leq g(p)$ e, portanto, $c \leq g(p)$. Logo, $g(p)=\max \{a \in A: f(a) \leq p\}$.

Proposição 23. Se $\left[f_{1}, g_{1}\right]$ é uma adjunção para $\left(A, \leq_{A}\right) e\left(B, \leq_{B}\right) e\left[f_{2}, g_{2}\right]$ é uma adjunção para $\left(B, \leq_{B}\right) e\left(C, \leq_{C}\right)$, então $\left[f_{2} \circ f_{1}, g_{1} \circ g_{2}\right]$ é uma adjunção para $\left(A, \leq_{A}\right) e$ $\left(C, \leq_{C}\right)$.

Demonstração. $a \leq g_{1} \circ g_{2}(c)=g_{1}\left(g_{2}(c)\right) \Leftrightarrow f_{1}(a) \leq g_{2}(c) \Leftrightarrow f_{2}\left(f_{1}(a)\right) \leq c \Leftrightarrow$ $f_{2} \circ f_{1}(a) \leq c$.

Cada particular par de Galois tem resultados semelhantes a estes sobre as adjunções.

Exemplo 7. Consideremos um espaço quase topológico $(E, \Omega)$ e observemos que $(\mathcal{P}(E), \subseteq)$ constitui um poset. Definindo as funções $f, g:(\mathcal{P}(E), \subseteq) \longrightarrow(\mathcal{P}(E), \subseteq)$ de modo que $f(A) \bar{A}$ e $g(A) \AA$ teremos que o par $[f, g]$ não constitui uma adjunção. Para mostrar a validade dessa afirmação, num primeiro momento, basta observar que, em geral, $g \circ f$ não é inflacionária, isto é, nem sempre vale que $A \subseteq g(f(A))=\stackrel{\circ}{A}$. $A$ afirmação de que o referido par não é uma adjunção decorre então da Proposição 16

\section{Introdução do axioma B na lógica TK}

Os espaços quase topológicos são modelos adequados, isto é, corretos e completos, para a lógica TK. O processo de demonstração dessa afirmação é bastante elaborado e daremos uma ideia do procedimento realçando a parte que nos interessa.

Nas definições que seguem consideramos um espaço quase topológico $(E, \Omega)$ arbitrário. 
Definição 18. $O$ conjunto $\operatorname{Var}(T K)=\left\{p_{1}, p_{2}, p_{3}, \ldots\right\}$, das variáveis proposicionais de $T K$, corresponde às fórmulas atômicas de $T K$.

Definição 19. $O$ conjunto das fórmulas de $T K$, denotado por For $(T K)$, é definido indutivamente por:

(i) cada variável $p_{i}$ está em For $(T K)$;

(ii) se $\varphi, \psi \in$ For $(T K)$, então $\varphi \rightarrow \psi \in$ For $(T K)$;

(iii) nada mais pertence a For $(T K)$.

Definição 20. Uma valoração restrita é uma função $\breve{v}: \operatorname{Var}(T K) \longrightarrow \mathcal{P}(E)$, que interpreta cada variável de $\mathbf{T K}$ em um elemento de $\mathcal{P}(E)$.

Definição 21. Uma valoração é uma função $v: F \operatorname{or}(T K) \longrightarrow \mathcal{P}(E)$ que estende $\breve{v}$, natural e unicamente, como segue:

(i) $v(p)=\breve{v}(p)$, para todo p pertencente a $\operatorname{Var}(T K)$;

(ii) $v(\neg \varphi)=E-v(\varphi)=v(\varphi)^{c}$;

(iii) $v(\diamond \varphi)=\overline{v(\varphi)}$;

(iv) $v(\varphi \wedge \psi)=v(\varphi) \cap v(\psi)$;

(v) $v(\varphi \vee \psi)=v(\varphi) \cup v(\psi)$;

(vi) $v(\top)=E$, em que $\top$ é qualquer tautologia;

(vii) $v(\perp)=\emptyset$, em que $\perp$, alguma contradição.

Proposição 24. Dada uma valoração qualquer $v:$ For $(T K) \longrightarrow \mathcal{P}(E)$ temos que $v(\diamond \varphi)=v(\varphi)$.

Demonstração. Decorre das definições de valoração, de $\diamond$, de fecho e de interior, observando ainda que $v(\diamond \varphi)=v(\neg \neg \varphi)$.

Definição 22. Dado $\Gamma \subseteq$ For $(\boldsymbol{T K})$, dizemos que uma valoração $v:$ For $(T K) \longrightarrow \mathcal{P}(E)$ é um modelo para $\Gamma$ se, para toda $\varphi \in \Gamma$, temos $v(\varphi)=E$.

Denotamos por $\langle(E, \Omega), v\rangle \models \Gamma$ o fato de $v$ ser um modelo para $\Gamma$ em $(E, \Omega)$. Em particular, se $\Gamma=\{\varphi\}$, então uma valoração $v$ é modelo para $\Gamma$, ou modelo para $\varphi$, se $v(\varphi)=E$. Neste caso escrevemos simplesmente $\langle(E, \Omega), v\rangle \models \varphi$ e dizemos que a fórmula $\varphi$ é verdadeira em $(E, \Omega)$.

Definição 23. Uma fórmula $\varphi \in$ For $(\boldsymbol{T K})$ é válida, o que é denotado por $\models \varphi$, se para todo espaço quase topológico $(E, \Omega)$ e toda valoração $v: F$ or $(T K) \longrightarrow(E, \Omega)$ temos que $\langle(E, \Omega), v\rangle \models \varphi$, isto é, que $v(\varphi)=E$.

Definição 24. Um subconjunto $\Gamma \subseteq$ For $(\boldsymbol{T K})$ implica semanticamente $\varphi$, o que é denotado por $\Gamma \models \varphi$, se todo modelo de $\Gamma$ é também modelo de $\varphi$. 
Com essas definições podemos enunciar os teoremas de correção e completude que caracterizam a adequação.

Teorema 1. (Correção) Se $\Gamma \vdash \varphi$, então $\Gamma \models \varphi$.

Note-se que, no teorema acima, o símbolo $\Gamma \vdash \varphi$ tem o significado usual de $\varphi$ ser consequência sintática ou dedutiva de $\Gamma$.

Para demonstrarmos a Completude, isto é, que toda consequência semântica implica na consequência sintática correspondente, o processo é mais elaborado.

Para maiores detalhes, ver [4].

Consideraremos agora a inclusão, na lógica TK, de um outro axioma, bastante conhecido no estudo das lógicas modais, chamado de Axioma B: $\varphi \rightarrow \diamond \diamond \varphi$.

Para o que seguirá é interessante notar que este axioma $\mathbf{B}$ pode ser expresso em uma forma equivalente que é: $\diamond \downarrow \varphi \rightarrow \varphi$. De fato, acompanhemos as equivalências abaixo:

$$
\begin{aligned}
& \diamond(\neg \varphi) \rightarrow(\neg \varphi) \Leftrightarrow \neg \nabla \neg \nabla(\neg \varphi) \rightarrow \neg \varphi \Leftrightarrow \varphi \rightarrow \diamond \neg \nabla(\neg \varphi) \Leftrightarrow \varphi \rightarrow \diamond \neg(\neg \vee \neg(\neg \varphi)) \\
\Leftrightarrow & \varphi \rightarrow \diamond \vee \varphi .
\end{aligned}
$$

\section{Oxioma B e uma adjunção nos espaços quase topoló- gicos}

Na medida em que consideramos a lógica TK com a inclusão do Axioma $\mathbf{B}$, devido à adequação, teremos no espaço quase topológico associado $(E, \Omega)$ uma corresponde restrição.

Assim é que a inclusão, como axioma, de $\varphi \rightarrow \diamond \downarrow$ exigirá, necessariamente, que para toda $\varphi \in \operatorname{For}(\mathbf{T K})$ tenhamos $v(\varphi \rightarrow \diamond \vee \varphi)=E$.

Acompanhemos as considerações abaixo para ver o que isso acarreta.

$v(\varphi \rightarrow \diamond \vee \varphi)=E \Leftrightarrow v(\neg \varphi \vee \diamond \diamond \varphi)=E \Leftrightarrow v(\neg \varphi) \cup v(\diamond \vee \varphi)=E \Leftrightarrow v(\varphi)^{c} \cup$ $v(\stackrel{\bullet}{\varphi})=E \Leftrightarrow v(\varphi)^{c} \cup \frac{\circ}{v(\varphi)}=E$.

Decorre que, para toda $\varphi \in \operatorname{For}(\mathbf{T K})$, deveremos ter $v(\varphi) \subseteq \overline{v(\varphi)}$.

Tendo em vista a equivalência $\varphi \rightarrow \diamond \diamond \varphi \Leftrightarrow \diamond \varphi \rightarrow \varphi$, também será necessário que:

$$
v(\diamond \varphi \rightarrow \varphi)=v(\neg \diamond \varphi \vee \varphi)=v(\neg \diamond \vee \varphi) \cup v(\varphi)=\overline{v(\varphi)}^{c} \cup v(\varphi)=E .
$$


Assim, $v(\varphi)^{c} \subseteq \overline{v(\varphi)}^{c}$, resultando que, para toda $\varphi \in$ For $(\mathbf{T K})$, deveremos ter $\overline{v(\varphi)} \subseteq v(\varphi)$.

Essas considerações podem ser resumidas no seguinte Lema:

Lema 1. Se o espaço quase topológicos $(E, \Omega)$ está associado à lógica $\mathbf{T K}$, munida com o axioma $\boldsymbol{B}$, então para todo $A \subseteq E$ são válidas:

(i) $A \subseteq \stackrel{\circ}{A}$;

(ii) $\bar{\AA} \subseteq A$.

Demonstração. Seja $(E, \Omega)$ um modelo para $\mathbf{T K}+\mathbf{B}$. Então $(E, \Omega)$ é um espaço quasetopológico e, portanto, de Tarski que modela também o axioma $\mathbf{B}$ e sua versão dual $\diamond \varphi \rightarrow \varphi$.

Assim, como cada fórmula $\varphi$ de TK é interpretada num subconjunto de $E$, se $v(\varphi)=$ $A$, das considerações acima, temos que $A \subseteq \stackrel{\circ}{\bar{A}}$ e $\bar{A} \subseteq A$.

Notemos inicialmente que todo espaço topológico é um espaço quase topológico e, portanto, satisfaz todos os axiomas da lógica TK. No entanto, o espaço topológico usual da reta não satisfaz as condições do Lema (1). Adicionalmente, todo espaço clopen é modelo de $\mathbf{T K}+\mathbf{B}$.

Voltemos agora à situação do Exemplo 7, respeitando as considerações originadas do processo que acabamos de constatar.

Proposição 25. Seja $(E, \Omega)$ um espaço quase topológico, modelo para uma lógica TK acrescida do axioma $\boldsymbol{B}$. Se $f, g:(\mathcal{P}(E), \subseteq) \rightarrow(\mathcal{P}(E), \subseteq)$ são funções definidas por: $f(A)={ }_{d f} \bar{A}$ e $g(A)=_{d f} \AA$, então o par $[f, g]$ constitui uma adjunção sobre $(E, \Omega)$.

Demonstração. Temos que os itens (iii) e (iv), da Proposição 16, são consequências diretas da Proposição 5. Já os itens (i) e (ii) da Proposição 16, decorrem do Lema 1 .

\section{Considerações Finais}

Neste trabalho identificamos e apresentamos, a partir da lógica TK acrescida do axioma B, um ambiente semântico adequado em que os operadores de fecho e interior de um espaço quase topológico constituem um par de Galois chamado adjunção. Os pares de Galois acabam por compôr um conceito que está presente em muitas situações da Lógica e da Matemática, por assim dizer eles ampliam o alcance da proposta inicial da conexão de Galois. 
Como temos muitos resultados conhecidos sobre os pares de Galois, podemos usálos para a exposição de muitos resultados sobre a Lógica TK acrescida do axioma $\mathbf{B}$, e também sobre os seus modelos de caráter topológico ou algébrico.

Assim, damos aqui os primeiros passos nesta inter-relação entre axiomas modais e seus respectivos modelos sobre TK. Uma questão interessante para ser abordada é o estudo da validade da recíproca da Proposição 25.

\section{Agradecimentos}

Agradecemos apoio da FAPESP e sugestões dos avaliadores.

\section{Referências}

[1] D’OTTAViAnO, I. M. L.; FEITOSA, H. A. Deductive systems and translations, In: BÉZIAU, J-Y.; LEITE, A. C.(Org.). Perspectives on universal logic. Monza: Polimetrica International Scientific Publisher, 2007. p. 125-157

[2] DUNN, J. M.; HARDEGREE, G. M. Algebraic methods in philosophical logic. Oxford: Oxford University Press, 2001.

[3] FEITOSA, H. A.; GRÁCIO, M. C. C.; NASCIMENTO, M. C. Logic TK: algebraic notions from Tarki's consequence operator. Principia, v. 14, p. 47-70, 2010.

[4] FEITOSA, H. A.; NASCIMENTO, M. C. Logic of deduction: models of pre-order and maximal theories. South American Journal of Logic, v. 1, p. 283-297, 2015.

[5] FEITOSA, H. A.; NASCIMENTO, M. C.; LAZARO, C. A. Pares de Galois e espaços de Tarski, Cognitio, v. 19, p. 110-132, 2018.

[6] HERRLICH, H.; HUSEK, M. Galois connections categorically. Journal of Pure and Applied Algebra, v. 68, p. 165-180, 1990.

[7] MIRAGLIA, F. Cálculo proposicional: uma interação da álgebra e da lógica. Campinas: UNICAMP/CLE, 1987. (Coleção CLE)

[8] NASCIMENTO, M. C.; FEITOSA, H. A. As álgebras dos operadores de conseqüência. Revista de Matemática e Estatística, v. 23, n. 1, p. 19-30, 2005.

[9] ORE, O. Galois connections. Transactions of the American Mathematical Society, v. 55, p. 493-513, 1944. 
[10] ORLOWSKA, E.; REWITZKY, I. Algebras for Galois-style connections and their discrete duality. Fuzzy Sets and Systems, v. 161, p. 1325-1342, 2010.

[11] SMITH, P. The Galois connection between syntax and semantics. Technical report. Cambridge: Univisity of Cambridge, 2010.

Aceito em 20 out. 2020 